\title{
EXISTENCE AND ASYMPTOTIC BEHAVIOR FOR SOME DIFFERENCE EQUATIONS ASSOCIATED WITH ACCRETIVE OPERATORS
}

\author{
N. C. Apreutesei \\ Department of Mathematics \\ Technical University of Iasi \\ 11, Bd. Copou, 6600, Iasi, Romania \\ ndumitri@tuiasi.ro
}

\begin{abstract}
We establish the existence, uniqueness and asymptotic behavior of the solution to a class of difference equations in a real Banach space, namely (1.1) below. The operator $A$ which governs the problem is m-accretive. This equation is of interest because it is the discrete analog of a class of evolution equations studied by many mathematicians.
\end{abstract}

Keywords: Accretive operator, m-accretive operator, strongly accretive operator, strongly monotone duality mapping.

\section{Introduction}

We are concerned with the difference equation

$$
\left\{\begin{array}{c}
u_{i+1}-\left(1+\theta_{i}\right) u_{i}+\theta_{i} u_{i-1} \in c_{i} A u_{i}, i \geq 1 \\
u_{0}=a, \quad \sup _{i \geq 1}\left\|u_{i}\right\|<\infty,
\end{array}\right.
$$

where $A$ is a nonlinear m-accretive (possibly multivalued) operator in a real Banach space $(X,\|\|),. a \in X$ is a given element, $\theta_{i} \geq 1, c_{i}>0$, $(\forall) i \geq 1$ are two given sequences of real numbers. The existence of the solution to (1.1) and some convergence properties of the solution are investigated in this paper.

G. Morosanu [12] established the existence and uniqueness of the solution for the boundary value problem

$$
\left\{\begin{array}{c}
u_{i+1}-2 u_{i}+u_{i-1} \in c_{i} A u_{i}, i \geq 1 \\
u_{0}=a, \quad \sup _{i \geq 0}\left\|u_{i}\right\|<\infty
\end{array}\right.
$$

The original version of this chapter was revised: The copyright line was incorrect. This has been corrected. The Erratum to this chapter is available at DOI: 10.1007/978-0-387-35690-7_44 
which corresponds to the case $\theta_{i} \equiv 1$ in (1.1). E. Mitidieri and G. Morosanu [11] analyzed the asymptotic behavior of the solution of problem (1.2) . Problem (1.2) was considered in a Hilbert space, where $A$ is a maximal monotone operator. It is the discrete analog of the boundary value problem

$$
\left\{\begin{array}{c}
u^{\prime \prime} \in A u, \quad t \in(0, \infty) \\
u(0)=a, \sup _{t \geq 0}\|u(t)\|<\infty,
\end{array}\right.
$$

which was studied by V. Barbu [7], [8]. The equation of problem (1.3) with the condition $u^{\prime}(0) \in \partial j(u(0)-a)$ instead of $u(0)=a$ (where function $j: H \rightarrow(-\infty,+\infty]$ is convex, lower-semicontinuous and proper and $\partial j$ is its subdifferential), was investigated by $\mathrm{H}$. Brézis [10] .

The existence and asymptotic behavior for (1.2) in the case when $A$ is an m-accretive operator in a Banach space, were proved by E. Poffald and S. Reich [14], [15] and by S. Reich and I. Shafrir [16] .

A generalization of equation (1.3) is

$$
p u^{\prime \prime}+r u^{\prime} \in A u+f, t \in(0, T) \quad(T \leq \infty) .
$$

In both cases $T<\infty$ and $T=\infty$, functions $p$ and $r$ are in $W^{1, \infty}(0, T)$. Papers concerned with this equation with different boundary conditions are due to L. Véron [17], N. Pavel [13], A. Aftabizadeh and N. Pavel [1], [2], N. Apreutesei [3], [4] , [5] .

A discretization of (1.4) with $f \equiv 0$ is

$$
p_{i}\left(u_{i+1}-2 u_{i}+u_{i-1}\right)+r_{i}\left(u_{i+1}-u_{i}\right) \in k_{i} A u_{i}, i \geq 1
$$

with $p_{i} \geq c>0, k_{i}>0,(\forall) i \geq 1$. Denoting by $\theta_{i}=\frac{p_{i}}{p_{i}+r_{i}}, c_{i}=\frac{k_{i}}{p_{i}+r_{i}}$, we find the equation of (1.1). Suppose that $\left(\theta_{i}\right)_{i \geq 1}$ is nonincreasing, $\theta_{i} \geq 1$, for all $i$. In Hilbert spaces, this equation was studied by $\mathrm{N}$. Apreutesei [6].

Recall some notions we need in the following sections.

Let $X$ be a real Banach space with the norm $\|\|,. X^{*}$ its dual space and $(.,$.$) the pairing between X$ and $X^{*}$. Denote by $J: X \rightarrow X^{*}$,

$$
J(x)=\left\{x^{*} \in X^{*},\left(x, x^{*}\right)=\|x\|^{2}=\left\|x^{*}\right\|^{2}\right\}
$$

the duality mapping of $X$. It is obvious that $J$ is monotone. $J$ is singlevalued if and only if $X$ is smooth. In this case we say that $J$ is strongly monotone if there is a positive constant $M$ such that

$$
(x-y, J x-J y) \geq M\|x-y\|^{2},(\forall) x, y \in X .
$$


A subset $A$ of $X \times X$ with the domain $D(A)$ and the range $R(A)$ is called accretive if for any $y_{i} \in A x_{i}, i=1,2$, there exists $j \in J\left(x_{1}-x_{2}\right)$ such that

$$
\left(y_{1}-y_{2}, j\right) \geq 0 \text {. }
$$

The accretive operator $A \subset X \times X$ is m-accretive if $R(I+A)=X$, where $I$ is the identity operator of $X$. It follows that $R(I+\lambda A)=X$, $(\forall) \lambda>0$. The operator $A \subset X \times X$ is said to be strongly accretive if there is a constant $\omega>0$ with the property: $(\forall) y_{i} \in A x_{i}, i=1,2,(\exists) j \in J\left(x_{1}-x_{2}\right)$, such that

$$
\left(y_{1}-y_{2}, j\right) \geq \omega\left\|x_{1}-x_{2}\right\|^{2} .
$$

It is known that if $A \subset X \times X$ is m-accretive, then $A$ is closed. If in addition $X^{*}$ is uniformly convex, then $A$ is demiclosed (strongly-weakly closed in $X \times X)$.

Section 2 is dedicated to the existence of the solution to problem (1.1). First we establish the existence for an auxiliary finite difference equation and then we use this result to the study of the problem (1.1). If $X$ has a strongly monotone duality mapping, then (1.1) has a unique solution, for any $a \in X$. Section 3 is concerned with the asymptotic behavior of the solution. First we give a weak convergence theorem. The Hilbert spaces case is separately studied. Finally we establish a strong convergence result under the hypothesis that $A$ is m-accretive and strongly accretive.

\section{Existence results}

In this section we shall study the existence and uniqueness of the solution for the difference inclusion (1.1).

We begin with an existence result for the auxiliary problem

$$
\left\{\begin{array}{c}
u_{i+1}-\left(1+\theta_{i}\right) u_{i}+\theta_{i} u_{i-1} \in c_{i} A u_{i}+f_{i}, 1 \leq i \leq N \\
u_{0}=a, \quad u_{N+1}=b,
\end{array}\right.
$$

where $N$ is a positive integer, $c_{i}>0, \theta_{i} \geq 1$ and $f_{i} \in X, 1 \leq i \leq N$. We work in the product space $X^{N}=X \times \ldots \times X$ provided with the norm

$$
|u|=\left(\sum_{i=1}^{N}\left\|u_{i}\right\|^{2}\right)^{1 / 2},(\forall) u=\left(u_{1}, \ldots, u_{N}\right) \in X^{N} .
$$

If $u=\left(u_{1}, \ldots, u_{N}\right) \in X^{N}$ and $u^{*}=\left(u_{1}^{*}, \ldots, u_{N}^{*}\right) \in\left(X^{*}\right)^{N}$, then we denote

$$
\left(u, u^{*}\right)_{N}=\sum_{i=1}^{N}\left(u_{i}, u_{i}^{*}\right)
$$


Theorem 2.1. If $X$ is a Banach space, $A \subset X \times X$ is an m-accretive operator, $\left(\theta_{i}\right)_{1<i<N}$ a nonincreasing sequence, $\theta_{i}>0, c_{i}>0,1 \leq i \leq N, a, b$ are in $X$ and $\left(f_{i}\right)_{1 \leq i \leq N} \in X^{N}$, then (2.1) has a unique solution in $X^{N}$. Proof. Denote by $\mathcal{A} \subset X^{N} \times X^{N}$ the operator

$$
\mathcal{A} u=\left\{\left(c_{1} v_{1}, \ldots, c_{N} v_{N}\right), v_{i} \in A u_{i}, 1 \leq i \leq N\right\}+\left(\theta_{1} a, 0, \ldots, 0, b\right),
$$

where $u=\left(u_{1}, \ldots, u_{N}\right) \in D(A)^{N}$ and by $B: X^{N} \rightarrow X^{N}$ the operator

$$
\begin{gathered}
B u=\left(\left(1+\theta_{1}\right) u_{1}-u_{2},-\theta_{2} u_{1}+\left(1+\theta_{2}\right) u_{2}-u_{3}, \ldots,\right. \\
\left.-\theta_{N-1} u_{N-2}+\left(1+\theta_{N-1}\right) u_{N-1}-u_{N},-\theta_{N} u_{N-1}+\left(1+\theta_{N}\right) u_{N}\right) .
\end{gathered}
$$

The operator $\mathcal{A}$ is m-accretive in $X^{N}$ and $B$ is continuous, everywhere defined and strongly accretive. Indeed, since $B$ is linear, to show the strong accretivity of $B$ it is sufficient to prove that $(\exists) \alpha>0$, such that

$$
\left(B u, u^{*}\right)_{N} \geq \alpha|u|^{2},(\forall) u \in X^{N},(\forall) u^{*} \in J u .
$$

But

$$
\left(B u, u^{*}\right)_{N}=\sum_{i=1}^{N}\left(1+\theta_{i}\right)\left(u_{i}, u_{i}^{*}\right)-\sum_{i=1}^{N-1}\left[\left(u_{i+1}, u_{i}^{*}\right)+\theta_{i+1}\left(u_{i}, u_{i+1}^{*}\right)\right],
$$

where $u_{i}^{*} \in J u_{i}, u_{i+1}^{*} \in J u_{i+1}$. Since $\left(\theta_{i}\right)_{1 \leq i \leq N}$ is nonincreasing, by $2\left(u_{i+1}, u_{i}^{*}\right) \leq \gamma_{i}\left\|u_{i+1}\right\|^{2}+\left(1 / \gamma_{i}\right)\left\|u_{i}\right\|^{2}, 2\left(u_{i}, u_{i+1}^{*}\right) \leq \gamma_{i}\left\|u_{i+1}\right\|^{2}+\left(1 / \gamma_{i}\right)\left\|u_{i}\right\|^{2}$, for all $\gamma_{i}>0$, we find

$$
\left(B u, u^{*}\right)_{N} \geq \sum_{i=1}^{N} \alpha_{i}\left\|u_{i}\right\|^{2}
$$

with

$$
\left\{\begin{array}{l}
\alpha_{1}=1+\theta_{1}-\frac{1}{2 \gamma_{1}}\left(1+\theta_{2}\right) \\
\alpha_{i}=1+\theta_{i}-\frac{1}{2 \gamma_{i}}\left(1+\theta_{i+1}\right)-\frac{\gamma_{i-1}}{2}\left(1+\theta_{i}\right), 2 \leq i \leq N-1 \\
\alpha_{N}=\left(1+\theta_{N}\right)\left(1-\frac{\gamma_{N-1}}{2}\right) .
\end{array}\right.
$$

Taking, for example $\gamma_{i}=i(i+2) /(i+1)^{2}$, we get $\alpha_{i}>0,1 \leq i \leq N$. Denoting $\alpha=\min \left\{\alpha_{i}, 1 \leq i \leq N\right\}>0$, from (2.5) one deduces

$$
\left(B u, u^{*}\right)_{N} \geq \alpha|u|^{2}
$$


i.e. $B$ is strongly accretive. This implies that $\mathcal{A}+B$ is $\mathrm{m}$-accretive and coercive, and consequently surjective: $R(\mathcal{A}+B)=X^{N}$. This means that problem (2.1) has a solution. It is easy to show the uniqueness of the solution.

Now we are going to study the difference equation (1.1), supposing that $X$ has a strongly monotone duality mapping $J$. For $\theta_{i} \equiv 1$, this problem was studied by G. Morosanu [12] in Hilbert spaces and by E. Poffald and S. Reich [14] in Banach spaces. Recall that $J$ is strongly monotone if and only if $X$ is uniformly convex with a modulus of convexity of power type 2 ([14]).

We state the following existence and uniqueness result.

Theorem 2.2. Let $X$ be a Banach space with a strongly monotone duality mapping $J$ and $A \subset X \times X$ an m-accretive operator, with $0 \in R(A)$. Let $\left(c_{i}\right)_{i \geq 1},\left(\theta_{i}\right)_{i>1}$ be two sequences, $c_{i}>0, \theta_{i} \geq 1,(\forall) i \geq 1, \theta_{i}$ nonincreasing. Then, for every $a \in X$, problem (1.1) has a unique solution $\left(u_{i}\right)_{i \geq 1}$, with $u_{i} \in D(A)$ for all $i \geq 1$.

Proof. By Theorem 2.1, the sequence of approximating problems

$$
\left\{\begin{array}{c}
u_{i+1}^{N}-\left(1+\theta_{i}\right) u_{i}^{N}+\theta_{i} u_{i-1}^{N} \in c_{i} A u_{i}^{N}, 1 \leq i \leq N \\
u_{0}^{N}=u_{N+1}^{N}=a
\end{array}\right.
$$

has a unique solution $\left(u_{i}^{N}\right)_{1 \leq i \leq N} \in X^{N}$.

For a given $w \in A^{-1}(0)$, we set $w_{i}^{N}=u_{i}^{N}-w, 0 \leq i \leq N+1$. By the accretivity of $A$, for every $i \in\{1, \ldots, N\}$, there is a $j_{i} \in J\left(w_{i}^{N}\right)$, such that

$$
\left(w_{i+1}^{N}-\left(1+\theta_{i}\right) w_{i}^{N}+\theta_{i} w_{i-1}^{N}, j_{i}\right) \geq 0 .
$$

This implies

$$
\left\|w_{i}^{N}\right\| \leq \frac{1}{1+\theta_{i}}\left\|w_{i+1}^{N}\right\|+\frac{\theta_{i}}{1+\theta_{i}}\left\|w_{i-1}^{N}\right\|, 1 \leq i \leq N
$$

hence

$$
\left\|w_{i}^{N}\right\|=\left\|u_{i}^{N}-w\right\| \leq \max \left(\left\|w_{0}^{N}\right\|,\left\|w_{N+1}^{N}\right\|\right)=\|a-w\|, 1 \leq i \leq N
$$

and thus

$$
\left\|u_{i}^{N}\right\| \leq\|w\|+\|a-w\|, 1 \leq i \leq N .
$$

We prove now the convergence of $u_{i}^{N}$ as $N \rightarrow \infty$ (uniformly for $i$ belonging to every finite set of natural numbers) to an element $u_{i}$ which verifies (1.1).

Let $N_{0}<N_{1}<N_{2}$ be positive integers and $v_{i}=u_{i}^{N_{2}}-u_{i}^{N_{1}}, 0 \leq i \leq$ $N_{1}+1$. Since $A$ is accretive, for each $i$ there is a $l_{i} \in J v_{i}$ such that

$$
\left(v_{i+1}-\left(1+\theta_{i}\right) v_{i}+\theta_{i} v_{i-1}, l_{i}\right) \geq 0 \text {. }
$$


Hence

$$
\left(v_{i+1}-v_{i}, l_{i}\right)-\theta_{i}\left(v_{i}-v_{i-1}, l_{i-1}\right) \geq \theta_{i}\left(v_{i}-v_{i-1}, l_{i}-l_{i-1}\right)
$$

and, since $J$ is strongly monotone (say of constant $M$ ), we get

$$
M \theta_{i}\left\|v_{i}-v_{i-1}\right\|^{2} \leq\left(v_{i+1}-v_{i}, l_{i}\right)-\theta_{i}\left(v_{i}-v_{i-1}, l_{i-1}\right),
$$

for $1 \leq i \leq N_{1}$. One multiplies (2.11) by $\theta_{k} \ldots \theta_{i+1}$ and one sums from $i=1$ to $i=k$, with $k \in\left\{1, \ldots, N_{1}\right\}$, to find

$$
M \sum_{i=1}^{k} \theta_{k} \ldots \theta_{i+1} \theta_{i}\left\|v_{i}-v_{i-1}\right\|^{2} \leq\left(v_{k+1}-v_{k}, l_{k}\right)-\theta_{k} \ldots \theta_{1}\left(v_{1}-v_{0}, l_{0}\right) .
$$

Since $l_{0}=0$ and $\theta_{i} \geq 1, i \geq 1$, it follows

$$
M \sum_{i=1}^{k}\left\|v_{i}-v_{i-1}\right\|^{2} \leq \frac{1}{2}\left(\left\|v_{k+1}\right\|^{2}-\left\|v_{k}\right\|^{2}\right), 1 \leq k \leq N_{1} .
$$

Since $\left\|v_{k}\right\|=\sum_{i=1}^{k}\left(\left\|v_{i}\right\|-\left\|v_{i-1}\right\|\right) \leq \sum_{i=1}^{k}\left\|v_{i}-v_{i-1}\right\|,(2.12)$ implies

$$
\left\|v_{k}\right\|^{2} \leq \frac{k}{2 M}\left(\left\|v_{k+1}\right\|^{2}-\left\|v_{k}\right\|^{2}\right) .
$$

We sum from $k=N_{0}$ to $k=N_{1}$, to get with the aid of (2.9)

$$
\sum_{k=N_{0}}^{N_{1}} \frac{1}{k}\left\|v_{k}\right\|^{2} \leq \frac{1}{2 M}\left(\left\|v_{N_{1}+1}\right\|^{2}-\left\|v_{N_{0}}\right\|^{2}\right) \leq C .
$$

By (2.13) we have $\left\|v_{k}\right\| \leq\left\|v_{k+1}\right\|$, for all $1 \leq k \leq N_{1}$. Hence for every $i \leq N_{0}$,

$$
\left\|v_{i}\right\|^{2}\left(\sum_{k=N_{0}}^{N_{1}} \frac{1}{k}\right) \leq \sum_{k=N_{0}}^{N_{1}} \frac{1}{k}\left\|v_{k}\right\|^{2} \leq C,
$$

so

$$
\left\|u_{i}^{N_{2}}-u_{i}^{N_{1}}\right\|^{2} \leq C /\left(\sum_{k=N_{0}}^{N_{1}} \frac{1}{k}\right), 1 \leq i \leq N_{0} .
$$

Therefore, there exists the limit $u_{i}=\lim _{N \rightarrow \infty} u_{i}^{N}$, for all $i$ belonging to every finite set of natural numbers. Since $A$ is m-accretive in $X$, we may pass to the limit in (2.7) to deduce that $\left(u_{i}\right)_{i \geq 1}$ is a solution of problem (1.1). The uniqueness follows easily in the same manner. 


\section{Asymptotic behavior}

In this section, we give some weak convergence and strong convergence results for the solution of the problem (1.1). Let's begin with an auxiliary result.

Proposition 3.1. Under the hypotheses of Theorem 2.2, if $\left(u_{i}\right)_{i \geq 1}$ is the unique solution of problem (1.1), then $(\forall) w \in A^{-1}(0)$, the sequence $\left(\left\|u_{i}-w\right\|\right)_{i \geq 1}$ is noincreasing. Moreover, there exists the limit

$$
h(w)=\lim _{i \rightarrow \infty}\left\|u_{i}-w\right\| .
$$

Proof. We pass to the limit as $N \rightarrow \infty$ in (2.8), where $w_{i}^{N}=u_{i}^{N}-w$, $1 \leq i \leq N$. Since the limit $u_{i}=\lim _{N \rightarrow \infty} u_{i}^{N}$ exists uniformly for $i$ belonging to every finite set of natural numbers, we get

$$
\left\|u_{i}-w\right\| \leq \frac{1}{1+\theta_{i}}\left\|u_{i+1}-w\right\|+\frac{\theta_{i}}{1+\theta_{i}}\left\|u_{i-1}-w\right\|, i \geq 1 .
$$

Since $\left(u_{i}\right)_{i>1}$ is bounded, this inequality shows that $\left(\left\|u_{i}-w\right\|\right)_{i>1}$ is nonincreasing. The monotonicity and the boundedness of $\left\|u_{i}-w\right\|$ lead us to the conclusion that the limit (3.1) exists.

Definition 3.1. The multivalued operator $A \subset X \times X$ is said to be injectiv if $A x_{1} \cap A x_{2} \neq \Phi$ implies $x_{1}=x_{2}$.

We state now a weak convergence theorem.

Theorem 3.1. Let $X$ be a Banach space with a strongly monotone duality mapping $J$ and let its dual $X^{*}$ be uniformly convex. Suppose $a \in X$ is a given element and $A \subset X \times X$ is injectiv and m-accretive, $0 \in R(A)$. Let $\left(c_{i}\right)_{i \geq 1},\left(\theta_{i}\right)_{i \geq 1}$ be like in Theorem $2.2, \theta_{1} \ldots \theta i / c_{i} \leq K$, $(\forall) i \geq 1$ ( $K$ is a positive constant). Then, the solution $\left(u_{i}\right)_{i \geq 1}$ of problem (1.1) converges weakly as $i \rightarrow \infty$ to a zero of $A$.

Proof. Denoting by $\left(\alpha_{i}\right)_{i>1}$ the sequence $\alpha_{0}=1, \alpha_{i}=1 / \theta_{1} \ldots \theta_{i},(\forall)$ $i \geq 1$, observe that $\alpha_{i-1} / \alpha_{i}=\theta_{i}$, so

$$
u_{i+1}-\left(1+\theta_{i}\right) u_{i}+\theta_{i} u_{i-1}=\frac{1}{\alpha_{i}}\left[\alpha_{i}\left(u_{i+1}-u_{i}\right)-\alpha_{i-1}\left(u_{i}-u_{i-1}\right)\right] .
$$

Thus problem (1.1) can be written as

$$
\left\{\begin{array}{c}
\frac{1}{\alpha_{i} c_{i}}\left(\varphi_{i+1}-\varphi_{i}\right) \in A u_{i}, i \geq 1 \\
u_{0}=a, \quad \sup _{i \geq 1}\left\|u_{i}\right\|<\infty
\end{array}\right.
$$


where $\varphi_{i}=\alpha_{i-1}\left(u_{i}-u_{i-1}\right)=\alpha_{i-1}\left(w_{i}-w_{i-1}\right)$. Here $w_{i}=u_{i}-w$, with a fixed $w \in A^{-1}(0)$. Since $A$ is accretive, for every $i$, there is $j_{i} \in J w_{i}$ such as

$$
\left(w_{i+1}-\left(1+\theta_{i}\right) w_{i}+\theta_{i} w_{i-1}, j_{i}\right) \geq 0 .
$$

This is equivalent with

$$
\begin{gathered}
\frac{1}{\alpha_{i}}\left[\left(\varphi_{i+1}, j_{i}\right)-\left(\varphi_{i}, j_{i-1}\right)\right] \geq \frac{1}{\alpha_{i}}\left(\varphi_{i}, j_{i}-j_{i-1}\right)= \\
=\frac{\alpha_{i-1}}{\alpha_{i}}\left(u_{i}-u_{i-1}, j_{i}-j_{i-1}\right)
\end{gathered}
$$

and since $J$ is strongly accretive (of constant $M$ ), for every positive integer $N$, we get

$$
\begin{aligned}
& M \sum_{i=1}^{N} \alpha_{i-1}\left\|u_{i}-u_{i-1}\right\|^{2} \leq \sum_{i=1}^{N}\left[\left(\varphi_{i+1}, j_{i}\right)-\left(\varphi_{i}, j_{i-1}\right)\right]= \\
& \quad=\frac{\alpha_{N}}{2}\left(\left\|w_{N+1}\right\|^{2}-\left\|w_{N}\right\|^{2}\right)+\frac{1}{2}\left\|w_{1}\right\|^{2}+\frac{3}{2}\left\|w_{0}\right\|^{2} .
\end{aligned}
$$

Using the estimation for $w_{i}$ in the proof of Theorem 2.2, one obtains

$$
M \sum_{i=1}^{N} \alpha_{i-1}\left\|u_{i}-u_{i-1}\right\|^{2} \leq \frac{5}{2}\|a-w\|^{2} .
$$

Passing to the limit as $N \rightarrow \infty$, one finds that $\alpha_{i-1}\left\|u_{i}-u_{i-1}\right\|^{2} \rightarrow 0$. Since $\alpha_{i-1} \leq 1$, we have $\alpha_{i-1}\left(u_{i}-u_{i-1}\right) \rightarrow 0$ strongly in $X$.

Let $u$ be a weak limit of a weakly convergent subsequence $\left(u_{i_{n}}\right)$ of $\left(u_{i}\right)_{i \geq 1}$. By the assumption $\theta_{1} \ldots \theta_{i} / c_{i} \leq K,(\forall) i \geq 1$, since $A$ is demiclosed, we may pass to the limit in (3.3) (as $i_{n} \rightarrow \infty$ ) and obtain $0 \in A u$.

If $u, \widetilde{u}$ are two such weak limits of some weakly convergent subsequences of $\left(u_{i}\right)_{i>1}$, then from the injectivity of $A$, we deduce that $u=\widetilde{u}$. So there is an element $u \in A^{-1}(0)$ such that $u_{i} \rightarrow u, i \rightarrow \infty$.

In Hilbert spaces, we have the following result, which for $\theta_{i} \equiv 1$ was proved by G. Morosanu [12].

Theorem 3.2. Let $H$ be a real Hilbert space, $a \in H$ be given and $A: D(A) \subset H \rightarrow H$ be a maximal monotone operator in $H$, with $0 \in R(A)$. If $\left(c_{i}\right)_{i \geq 1},\left(\theta_{i}\right)_{i \geq 1}$ satisfy the conditions of Theorem 3.1 and, in addition, there is a constant $c>0$ such that $c_{i} \geq c>0$, then the solution $u_{i}$ of problem (1.1) is weakly convergent to a zero of $A$. 
We give now a strongly convergence result. The proof is similar to the Hilbert spaces case (see N. Apreutesei [6]).

Theorem 3.3. Let $X$ be a Banach space with a strongly monotone duality mapping $J, a \in X$ be a given element and $A \subset X \times X$ an $m$-accretive, strongly accretive and univoque operator with $0 \in R(A)$. Let $\left(c_{i}\right)_{i \geq 1},\left(\theta_{i}\right)_{i \geq 1}$ be two sequences such that $\left(\theta_{i}\right)_{i \geq 1}$ is nonincreasing, $c_{i}>0, \theta_{i} \geq 1,(\forall) i \geq 1$ and $\sum_{i=1}^{\infty} c_{i} / \theta_{i}=\infty$. Then, the solution $\left(u_{i}\right)_{i \geq 1}$ of problem (1.1) is strongly convergent as $i \rightarrow \infty$ to the only element of $A^{-1} 0$.

\section{References}

[1] A.R. Aftabizadeh and N.H. Pavel, Boundary value problems for second order differential equations and a convex problem of Bolza, Diff. Integral Eqns. 2(1989), 495-509.

[2] A.R. Aftabizadeh and N.H. Pavel, Nonlinear boundary value problems for some ordinary and partial differential equations associated with monotone operators, J.Math.Anal.Appl.156(1991),535-557.

[3] N.C. Apreutesei, A boundary value problem for second order differential equations in Hilbert spaces, Nonlinear Analysis, TMA, 24(1995), 1235-1246.

[4] N.C. Apreutesei, Some second order evolution equations governed by maximal monotone operators, Anal. Univ. Craiova, 24(1997), 45-61.

[5] N.C. Apreutesei, Second order differential equations on half-line associated with monotone operators, J.Math.Anal.Appl.223(1998), 472-493.

[6] N.C. Apreutesei, Existence and asymptotic behavior for a class of second order difference equations, to appear in J. Difference Eq. Appl.

[7] V. Barbu, Sur un probleme aux limites pour une classe d'equations differentielles nonlineaires abstraites du deuxieme ordre en t, C.R.Acad.Sci. Paris 274(1972), 459-462.

[8] V. Barbu, A class of boundary problems for second order abstract differential equations, J.Fac.Sci.Univ.Tokyo,Sect 1, 19(1972), 295-319.

[9] V. Barbu, Nonlinear Semigroups and Differential Equations in Banach Spaces, Noordhoff, Leyden, 1976.

[10] H. Brézis, Equations d'evolution du second ordre associees a des operateurs monotones, Israel J. Math.12(1972), 51-60.

[11] E. Mitidieri and G. Moroşanu, Asymptotic behaviour of the solutions of second order difference equations associated to monotone operators, Numerical Funct. Anal.Optim. 8(1986-1987), 419-434.

[12] G. Moroşanu, Second order difference equations of monotone type, Numerical Funct. Anal.Optim.1(1979), 441-450.

[13] N. Pavel, Nonlinear boundary value problems for second order differential equations, J. Math. Anal. Appl. 50(1975) 373-383. 
[14] E. Poffald and S. Reich, An incomplete Cauchy problem, J. Math. Anal. Appl., 113(1986), 514-543.

[15] E. Poffald and S. Reich, A difference inclusion, in "Nonlinear Semigroups, Partial Differential Equations and Attractors", Lecture Notes in Mathematics, vol. 1394, Springer, Berlin, 1989, 122-130.

[16] S. Reich and I. Shafrir, An existence theorem for a difference inclusion in general Banach spaces, J. Math. Anal. Appl., 160 (1991), 406-412.

[17] L. Véron, Problèmes d'évolution du second ordre associés à des opérateurs monotones, C.R. Acad.Sci.Paris 278(1974), 1099-1101. 